\title{
Hamiltonian Systems with Widely Separated Frequencies
}

\author{
J.M. Tuwankotta* \\ F. Verhulst \\ e-mail: tuwankotta@math.u1.nl, verhulst@math.u1.nl. \\ Mathematisch Instituut, Utrecht University \\ PO Box 80.010, 3508 TA Utrecht \\ The Netherlands
}

\begin{abstract}
In this paper we study two degree of freedom Hamiltonian systems and applications to nonlinear wave equations. Near the origin, we assume that near the linearized system has purely imaginary eigenvalues: $\pm i \omega_{1}$ and $\pm i \omega_{2}$, with $0<\omega_{2} / \omega_{1} \ll 1$ or $\omega_{2} / \omega_{1} \gg 1$, which is interpreted as a perturbation of a problem with double zero eigenvalues. Using the averaging method, we compute the normal form and show that the dynamics differs from the usual one for Hamiltonian systems at higher order resonances. Under certain conditions, the normal form is degenerate which forces us to normalize to higher degree. The asymptotic character of the normal form and the corresponding invariant tori is validated using KAM theorem. This analysis is then applied to widely separated mode-interaction in a family of nonlinear wave equations containing various degeneracies.
\end{abstract}

Keywords. Hamiltonian mechanics, resonance, normal forms, widely separated frequencies.

\section{Introduction}

The dynamics of two degrees of freedom Hamiltonian systems near stable equilibrium is relatively well understood; see for instance [3] pp.258-270, [11] pp.212-226. Resonance is known to play an important role in the dynamics of a system of differential equations. The presence of resonance in a system significantly changes the behavior of the system. Consider for instance the flow on a torus with irrational slope - which corresponds to the

*On leave from Jurusan Matematika, FMIPA, Institut Teknologi Bandung, Ganesha no. 10, Bandung, Indonesia 
non-resonant case - compared to the flow on a torus with rational slope. Orbits of the system in the first case are dense while in the second case, all solutions are periodic.

In two degrees of freedom Hamiltonian systems, one can divide the resonances into three classes, namely first order resonances (also known as Fermi resonances), second order resonances, and higher order resonances (see [24] pp.146-162 for details). For systems in first order resonance, it is known that they may display parametric excitation. This behavior is characterized by energy transfer between the degrees of freedom. This energy transfer is already apparent on a relatively short time-scale for almost all solutions (see [26]). However, the presence of a discrete symmetry may change the situation (see for instance $[22,27])$. The higher order resonances also show some energy exchange but on a much smaller scale and on a much longer time-scale (see [23, 27]).

In this paper, we consider two degrees of freedom Hamiltonian systems with widely separated frequencies: the ratio between the frequencies is either very small or very large. The small parameter $\varepsilon$ is introduced into the system by rescaling the variables in the usual way. Such a system can be seen as a Hamiltonian system at an extreme high order resonance.

One might expect that if the natural frequency ratio is $1: \varepsilon$, then the system behaves like a non-resonant two degrees of freedom Hamiltonian system. We show in this paper that this assumption produces somewhat different phenomena than expected. The phasespace of a non-resonant Hamiltonian system near the origin is foliated by invariant tori. These tori persist (by KAM theorem) under a Hamiltonian perturbation. In the case of widely separated frequencies, the phase space is nearly filled up with unbounded solutions, except for a very small domain near the elliptic equilibrium point.

For $\varepsilon=0$ the system is linear with double zero eigenvalues of the equilibrium at the origin. Broer et. al. in [5] (or in [6] for more explanation), studied this class of Hamiltonian systems in a more general setting. These systems can be divided into two cases, i.e. the semi-simple case and the non semi-simple case. Using normal form theory and singularity theory, the above authors give a bifurcation analysis near the equilibrium. The codimension of the equilibrium point is 1 for the non semi-simple case and 3 for the semisimple case. Their paper also describes the universal unfolding of the equilibrium point. In our paper we consider only the semi-simple case. We extend the normal form analysis of [5] by considering a number of possible degeneracies arising in applications. We are also interested in describing the dynamics (in time) of the system which, in a sense, also supplements the analysis done in $[5]$ or $[6]$.

In applications, this type of problem arises quite naturally. For instance in the analysis of a model for atmospheric ultra-low frequency variability in [9], the author found a case where one of the natural frequencies in the system is as small as the nonlinear terms. However, there the system is not Hamiltonian. Nayfeh et. al. [19], [20] and Haller [12] treat comparable cases in mechanical engineering. For a recent result, see also Langford and Zhan in $[16,17]$. We shall return to such problems in a separate paper.

Lower order resonances produce more spectacular dynamics but higher order resonances 
appear more frequently in applications. For instance in wave equations, cases where the resonances are of the type that we consider in this paper are quite natural. This fact also motivated our study of this type of Hamiltonian system.

In Section 2 we formulate our problem as one where perturbation theory and normal forms can be applied to approximate the full system. There are several ways to normalize a system of differential equations, namely using Lie-series, averaging, or using a generating function. For details on normalization using Lie-series, see [7, 8, 10], while for averaging or using a generating function see [2, 24].

We use the averaging method to compute the normal form. To verify the asymptotic nature of the normal form, the theory of averaging requires that we restrict ourselves to a domain of bounded solutions. For this, we approximate the locations of the saddle type equilibria of the system. The distance between these saddle points (if they exist) to the origin gives an indication of how large the domain of bounded solutions is. This is done in Section 3. We continue with the normal form computation to analyze the truncated normal form in Section 4 and 5. For some values of the parameters we have a degeneracy in the normal form, related to symmetry, which forces us to normalize to second-order. This situation is analyzed in Section 6 where we still find some nontrivial dynamics. We note that the assumption of the natural frequency being $O(\varepsilon)$ affects the domain of bounded solutions. Keeping this in mind, we use KAM theory to show the validity of the normal form in Section 7. In Section 8 we discuss systems with widely separated frequencies which arise from the spectrum of evolution operators with initial-boundary conditions. Examples of such systems can be found in conservative nonlinear wave equations. It is shown that although there is no exchange of energy between the modes, there can be a strong phase interaction.

\section{Mathematical formulation of the problem}

Consider a two degrees of freedom Hamiltonian (potential) system with Hamiltonian

$$
\begin{aligned}
H= & \frac{1}{2}\left(p_{x}{ }^{2}+x^{2}\right)+\frac{1}{2} \varepsilon\left(p_{y}{ }^{2}+y^{2}\right)-\left(\frac{1}{3} a_{1} x^{3}+a_{2} x^{2} y+a_{3} x y^{2}+\frac{1}{3} a_{4} y^{3}\right) \\
& -\left(\frac{1}{4} b_{1} x^{4}+b_{2} x^{3} y+b_{3} x^{2} y^{2}+b_{4} x y^{3}+\frac{1}{4} b_{5} y^{4}\right)+O\left(\left\|\left(x, y, p_{x}, p_{y}\right)^{T}\right\|^{5}\right) .
\end{aligned}
$$

The Hamiltonian system is defined on $\mathbb{R}^{4}$ with coordinates $\left(x, y, p_{x}, p_{y}\right)$ and symplectic form $\mathrm{d} x \wedge \mathrm{d} p_{x}+\mathrm{d} y \wedge \mathrm{d} p_{y}$. We assume that $\varepsilon$ is a small parameter: $0<\varepsilon \ll 1$. We also assume that all of the constants $a_{j}, j=1, \ldots, 4$ and $b_{j}, j=1, \ldots, 5$ are $O(1)$ with respect to $\varepsilon$. It is easy to see that for all $\varepsilon>0$, the origin is an elliptic equilibrium. We re-scale the variables (and also time) using the small parameter to localize the system around the origin in the usual way $(x=\varepsilon \bar{x}, \ldots)$. The Hamiltonian (2.1) then becomes (we use the same notation for the rescaled variables and Hamiltonian)

$$
\begin{aligned}
H= & \frac{1}{2}\left(p_{x}{ }^{2}+x^{2}\right)+\frac{1}{2} \varepsilon\left(p_{y}{ }^{2}+y^{2}\right)-\varepsilon\left(\frac{1}{3} a_{1} x^{3}+a_{2} x^{2} y+a_{3} x y^{2}+\frac{1}{3} a_{4} y^{3}\right) \\
& -\varepsilon^{2}\left(\frac{1}{4} b_{1} x^{4}+b_{2} x^{3} y+b_{3} x^{2} y^{2}+b_{4} x y^{3}+\frac{1}{4} b_{5} y^{4}\right)+O\left(\varepsilon^{3}\right) .
\end{aligned}
$$


Thus, we have a Hamiltonian perturbation of two harmonic oscillators with additional assumption that the basic frequency ratio in the system is $1: \varepsilon$.

\section{Remark 2.1 Two types of systems with widely separated frequencies .}

Consider a Hamiltonian systems with Hamiltonian

$$
H=\frac{1}{2} \omega_{1}\left(p_{1}^{2}+q_{1}^{2}\right)+\frac{1}{2} \omega_{2}\left(p_{2}^{2}+q_{2}^{2}\right)+H_{r} .
$$

where $H_{r}$ is a polynomial with degree at least three. There are two possibilities for the system generated by (2.3) to have widely separated frequencies. One might encounter the situation where $\omega_{2}=O(\varepsilon)$ as in the case of Hamiltonian (2.1). By rescaling the variables the Hamiltonian becomes

$$
H=\frac{1}{2}\left(p_{1}{ }^{2}+q_{1}{ }^{2}\right)+\frac{1}{2} \varepsilon\left(p_{2}{ }^{2}+q_{2}{ }^{2}\right)+\varepsilon H_{3}+\varepsilon^{2} H_{4}+\ldots,
$$

where $H_{3}$ represents the cubic terms, $H_{4}$ the quartic. etc. We call this situation the first type of widely separated frequencies.

The other possibility arises when $\omega_{1}=O(1 / \varepsilon)$. By rescaling time (and also $\varepsilon$ ) we derive the Hamiltonian of the form

$$
H=\frac{1}{2}\left(p_{1}^{2}+q_{1}^{2}\right)+\frac{1}{2} \varepsilon\left(p_{2}^{2}+q_{2}^{2}\right)+\varepsilon H_{r} .
$$

In general, the Hamiltonian system derived from this Hamiltonian is still too complicated to analyze as all the nonlinear terms are of the same order. Thus, we localize around the origin by rescaling the variables. The asymptotic ordering in this case, however, is different from the one in $(2.2)$ as the nonlinear terms become $O\left(\varepsilon^{2}\right)$ :

$$
H=\frac{1}{2}\left(p_{1}^{2}+q_{1}^{2}\right)+\frac{1}{2} \varepsilon\left(p_{2}^{2}+q_{2}^{2}\right)+\varepsilon^{2} H_{3}+\varepsilon^{3} H_{4}+\ldots
$$

We call this situation the second type. In Section 5, we will return to the relation between the first and the second type of resonances.

In the unperturbed case, i.e. $\varepsilon=0$, all solutions of the equations of motion derived from (2.2) are periodic with period $2 \pi$. Those solutions are of the form

$$
\left(x, y, p_{x}, p_{y}\right)=\left(r_{\circ} \cos \left(t+\varphi_{\circ}\right), y_{\circ},-r_{\circ} \sin \left(t+\varphi_{\circ}\right), p_{y_{0}}\right),
$$

where $r_{0}, \varphi_{0}, y_{0}$, and $p_{y_{0}}$ are determined by the given initial conditions. Moreover, all points of the form $\left(0, y, 0, p_{y}\right) \in \mathbb{R}^{4}$ are critical corresponding with equilibria which is not a generic situation in Hamiltonian systems. We expect that most of these equilibria will be perturbed away when $\varepsilon \neq 0$. Consequently, most of the periodic solutions are also perturbed away.

We use the averaging method to compute the normal form of the equations of motion derived from (2.2). Details on the averaging method can be found in [24]. The analysis is then valid up to order $\varepsilon$ on the time-scale $1 / \varepsilon$ at first-order, to order $\varepsilon^{2}$ on the time-scale $1 / \varepsilon$ at second-order. Before carrying out the normal form computation we first look at the domain where the solutions are bounded. 


\section{Domain of bounded solutions}

The theory of averaging requires the solutions of both the averaged and the original equations to stay in the interior of a bounded domain, at least for some time. Only in that domain, the averaging theorem guarantees the asymptotic character of the approximations. Thus, the existence of this domain is important.

The equations of motion derived from (2.2) are

$$
\begin{aligned}
& \dot{x}=p_{x} \\
& \dot{p}_{x}=-x+\varepsilon\left(a_{1} x^{2}+2 a_{2} x y+a_{3} y^{2}\right)+\varepsilon^{2}\left(b_{1} x^{3}+3 b_{2} x^{2} y+2 b_{3} x y^{2}+b_{4} y^{3}\right) \\
& \dot{y}=\varepsilon p_{y} \\
& \dot{p}_{y}=\varepsilon\left(-y+a_{2} x^{2}+2 a_{3} x y+a_{4} y^{2}\right)+\varepsilon^{2}\left(b_{2} x^{3}+2 b_{3} x^{2} y+3 b_{4} x y^{2}+b_{5} y^{3}\right) .
\end{aligned}
$$

We will approximate the equilibria of system (3.1). To do that, we set $x=x_{0}+\varepsilon x_{1}+O\left(\varepsilon^{2}\right)$ and $y=y_{0}+O(\varepsilon)$. It is clear that $p_{x}=p_{y}=0$ at the equilibria. Substituting these into (3.1) we have two equilibria if $a_{4} \neq 0$, i.e. $\left(x, y, p_{x}, p_{y}\right)=(0,0,0,0)$ and $\left(-\varepsilon a_{3} / a_{4}{ }^{2}, 1 / a_{4}, 0,0\right)$ while if $a_{4}=0$ up to this approximation we have only one equilibrium, i.e. $(0,0,0,0)$.

Define the potential function of the Hamiltonian (2.2), i.e.

$$
\begin{aligned}
V(x, y)= & \frac{1}{2}\left(x^{2}+\varepsilon y^{2}\right)-\varepsilon\left(\frac{1}{3} a_{1} x^{3}+a_{2} x^{2} y+a_{3} x y^{2}+\frac{1}{3} a_{4} y^{3}\right) \\
& -\varepsilon^{2}\left(\frac{1}{4} b_{1} x^{4}+b_{2} x^{3} y+b_{3} x^{2} y^{2}+b_{4} x y^{3}+\frac{1}{4} b_{5} y^{4}\right)+O\left(\varepsilon^{3}\right) .
\end{aligned}
$$

It is an easy exercise - by checking the second derivatives of (3.2) - to derive the stability of those equilibria found above. We conclude that $(0,0,0,0)$ is a center point and in the case where $a_{4} \neq 0$ we have $\left(-\varepsilon a_{3} / a_{4}{ }^{2}, 1 / a_{4}, 0,0\right)$ is a saddle point.

The fact that we have a possibility of having a saddle point in an $O(\varepsilon)$-neighborhood of the center point implies the domain of bounded solutions to shrink in measure (at least as fast as $\varepsilon$ as $\varepsilon$ goes to zero). This is in contrast with the cases where all the natural frequencies are of the same order where the measure of the domain is independent of $\varepsilon$.

\section{Normal form computation}

Consider again the equations of motion in (3.1). The equations for $y$ and $p_{y}$ in (3.1) are already in the Lagrange standard form. Thus we need only to transform the first two equations in (3.1).

Putting $x=r \cos (t+\varphi)$ and $p_{x}=-r \sin (t+\varphi)$, the equations of motion (3.1) become

$$
\begin{aligned}
\dot{\varphi}= & -\frac{1}{r} \cos (t+\varphi)\left(\varepsilon\left\{a_{1} r^{2} \cos ^{2}(t+\varphi)+2 a_{2} r \cos (t+\varphi) y+a_{3} y^{2}\right\}\right. \\
& \left.+\varepsilon^{2}\left\{b_{1} r^{3} \cos ^{3}(t+\varphi)+3 b_{2} r^{2} \cos ^{2}(t+\varphi) y+2 b_{3} r \cos (t+\varphi) y^{2}+b_{4} y^{3}\right\}\right) \\
\dot{r}= & -\sin (t+\varphi)\left(\varepsilon\left\{a_{1} r^{2} \cos ^{2}(t+\varphi)+2 a_{2} r \cos (t+\varphi) y+a_{3} y^{2}\right\}\right. \\
& \left.+\varepsilon^{2}\left\{b_{1} r^{3} \cos ^{3}(t+\varphi)+3 b_{2} r^{2} \cos ^{2}(t+\varphi) y+2 b_{3} r \cos (t+\varphi) y^{2}+b_{4} y^{3}\right\}\right) \\
\dot{y}= & \varepsilon p_{y} \\
\dot{p}_{y}= & \varepsilon\left(-y+a_{2}(r \cos (t+\varphi))^{2}+2 a_{3} r \cos (t+\varphi) y+a_{4} y^{2}\right) \\
& +\varepsilon^{2}\left(b_{2}(r \cos (t+\varphi))^{3}+2 b_{3}(r \cos (t+\varphi))^{2} y+3 b_{4} r \cos (t+\varphi) y^{2}+b_{5} y^{3}\right) .
\end{aligned}
$$


The right hand side of (4.1) is $2 \pi$-periodic in $t$. We note that the transformation to $(\varphi, r)$ is not a symplectic transformation. However, the averaged equations of motion are equivalent to the Birkhoff normal form of the equations of motion of (2.2).

For some values of the parameters, first order averaging is not sufficient. For this reason we compute the normal form up to $O\left(\varepsilon^{3}\right)$ using second-order averaging. After applying the second-order averaging method to (2.2), we transform $I=\frac{1}{2} r^{2}, \psi=t+\varphi$. The averaged equations of motion then read

$$
\begin{aligned}
& \dot{\psi}=1-\varepsilon a_{2} y-\varepsilon^{2}\left(\left(\frac{5}{6} a_{1}^{2}+\frac{3}{4} b_{1}\right) I+\left(\frac{1}{2} a_{2}^{2}+a_{1} a_{3}+b_{3}\right) y^{2}\right) \\
& \dot{I}=0 \\
& \dot{y}=\varepsilon p_{y} \\
& \dot{p}_{y}=\varepsilon\left(-y+a_{4} y^{2}+a_{2} I\right)+\varepsilon^{2}\left(2\left(\frac{1}{2} a_{2}^{2}+a_{1} a_{3}+b_{3}\right) y I+\left(2 a_{3}^{2}+b_{5}\right) y^{3}\right),
\end{aligned}
$$

which is a Hamiltonian system with Hamiltonian

$$
\mathcal{H}=I+\varepsilon \mathcal{H}_{1}+\varepsilon^{2} \mathcal{H}_{2},
$$

where

$$
\begin{aligned}
& \mathcal{H}_{1}=\frac{1}{2}\left(y^{2}+p_{y}{ }^{2}\right)-a_{2} I y-\frac{1}{3} a_{4} y^{3} \\
& \mathcal{H}_{2}=\frac{1}{2}\left(\frac{5}{6} a_{1}{ }^{2}+\frac{3}{4} b_{1}\right) I^{2}+\left(\frac{1}{2} a_{2}{ }^{2}+a_{1} a_{3}+b_{3}\right) y^{2} I+\frac{1}{4}\left(2 a_{3}{ }^{2}+b_{5}\right) y^{4}
\end{aligned}
$$

This implies that the total energy $H$ can be approximated by $\mathcal{H}=I+\varepsilon \mathcal{H}_{1}+\varepsilon^{2} \mathcal{H}_{2}$.

As expected in such an extreme type of higher order resonance, the interaction between the two oscillators is weak in the sense that up to this approximation, there is no interchange of energy between the degrees of freedom. However, there is phase-interaction. In the next section we will first analyze the $O(\varepsilon)$-term of (4.2).

Remark 4.1 As mentioned above, the transformation carrying $\left(x, p_{x}\right)$ into $(r, \varphi)$ is not symplectic. Nevertheless, after averaging and transformation to coordinates $(I, \psi)$ we regained the symplectic structure. The symplectic form is $\mathrm{d} \psi \wedge \mathrm{d} I+\mathrm{d} y \wedge \mathrm{d} p_{y}$. In the literature the pair $(I, \psi)$ is known as symplectic polar coordinates.

Remark 4.2 It is interesting to note that $\{\mathcal{H}, I\}=0$ where $\{$,$\} is the Poisson bracket.$ The Hamiltonian (4.3) then can be viewed as a normalized $H$ with respect to the $S^{1}$-action defined by the flow of the unperturbed Hamiltonian, $X_{I}$. Dividing out this action from the system (or equivalently fixing the value of $I$ ) leads to a reduced system (for reduction see [8]) which corresponds with a Poincaré section for the flow $X_{\mathcal{H}}$. This Poincaré section is an approximation of a section of the original flow $X_{H}$. In contrast with the other cases in this family of Hamiltonian systems, - those in which the frequencies are of the same order - in this case the reduced space is $\mathbb{R}^{2}$ (for non flat reduced spaces, see for instance $[7,21])$.

Remark 4.3 In applications, symmetries arise naturally in a system. One can consider for instance the discrete symmetry $\phi_{x}:\left(x, y, p_{x}, p_{y}\right)^{T} \mapsto\left(-x, y,-p_{x}, p_{y}\right)^{T}$ or $\phi_{y}$ : 
$\left(x, y, p_{x}, p_{y}\right)^{T} \mapsto\left(x,-y, p_{x},-p_{y}\right)^{T}$. If a Hamiltonian system is invariant under a symmetry $\phi$ then we have $\phi^{*} H=H \circ \phi=H$. The symmetry $\phi_{x}$ and $\phi_{y}$ is symplectic (they preserve the symplectic form). In [7] it is proved that the normalization can be done such that the symmetry $\phi_{x}$ or $\phi_{y}$ is preserved.

For a $y$-symmetric Hamiltonian, i.e. $a_{2}=a_{4}=b_{2}=b_{4}=0$, the normal form (4.2) is degenerate up to $O(\varepsilon)$. However, a non-trivial dynamics is achieved as the second-order

terms are included. On the other hands, for an $x$-symmetric Hamiltonian the normal form (4.2) is non-degenerate.

\section{$5 \quad$ First order analysis of the averaged equations}

In this section we analyze the Hamiltonian system (4.2) up to order $\varepsilon$. What we mean is that we drop all terms of $O\left(\varepsilon^{2}\right)$, i.e.

$$
\begin{aligned}
\dot{\psi} & =1-\varepsilon a_{2} y \\
\dot{I} & =0 \\
\dot{y} & =\varepsilon p_{y} \\
\dot{p}_{y} & =\varepsilon\left(-y+a_{4} y^{2}+a_{2} I\right) .
\end{aligned}
$$

The solutions of system (5.1) approximate the solutions of system (4.1) to $O(\varepsilon)$ on the time-scale $1 / \varepsilon$. The result of this section agree with $[5,6]$.

It is clear that $I(t) \geq 0$ for all time and up to $O(\varepsilon)$, it is related to the original Hamiltonian through $\mathcal{H}=I+\varepsilon \mathcal{H}_{1}$. Instead of fixing the value of $\mathcal{H}$ we fix $I=I_{\circ} \in \mathbb{R}$. Thus, we are looking at the flow of Hamiltonian system (5.1), $X_{\mathcal{H}}$, restricted to the manifold $I=I_{\circ}$. The reduced system (after rescaling time to $\tau=\varepsilon t$ ) is

$$
\begin{aligned}
\frac{d y}{d \tau} & =p_{y} \\
\frac{d p_{y}}{d \tau} & =\left(-y+a_{4} y^{2}+a_{2} I_{\circ}\right),
\end{aligned}
$$

which is a Hamiltonian system with Hamiltonian (4.4). In this reduced system, we might expect to have none, one or two equilibria. See also Remark 4.2.

There are four (three independent) important parameters in the Hamiltonian system (5.2), namely $a_{4}, a_{2}, I_{\circ}$, and

$$
D=1-4 a_{2} a_{4} I_{\circ}
$$

As mentioned in the previous section, if $a_{4} \neq 0$, in the $\varepsilon$-neighborhood of the origin we have another equilibrium of the saddle type. This equilibrium does not exist if $a_{4}=0$. Furthermore, the reduced system (5.2) is degenerate if $a_{4}=0$ : it becomes a linear oscillator. Thus, for $a_{4}=0$ we need to include quadratic terms in the normal form. We will do this in the next section. In this section we assume $a_{4} \neq 0$. 
Let $I_{\circ}=0$, the flow $X_{I_{0}}$ degenerates to a point. As a consequence, the flow $X_{\mathcal{H}}$ in this case lives in a two dimensional manifold defined by $(0,0) \times \mathbb{R}^{2}$ (or just $\mathbb{R}^{2}$ ). Furthermore, for $I_{\circ}=0$, we have $D=1>0$. Thus, the dynamics in the manifold $(0,0) \times \mathbb{R}^{2}$ is the same as the dynamics of the reduced system for $D>0$.

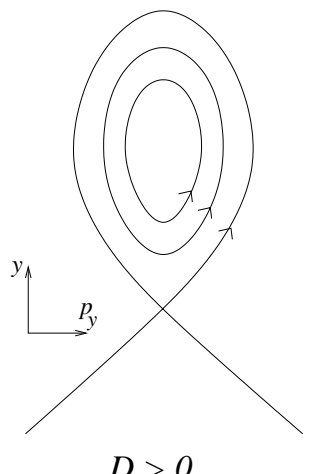

$D>0$

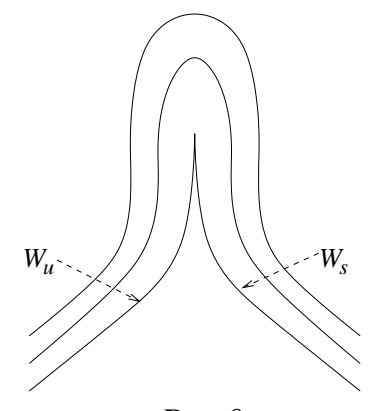

$D=0$

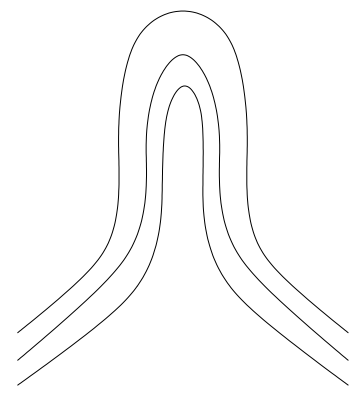

$D<0$

Figure 1: Dynamics of the Hamiltonian system (5.2) for $a_{4} \neq 0, I_{\circ} \neq 0$, and $a_{2} \neq 0$.

Let us assume that $I_{\circ}>0$. The flow $X_{I_{0}}$ defines a non-degenerate $S^{1}$-action on the phase-space of system (5.1). Thus, the flow $X_{\mathcal{H}}$ lives in $S^{1} \times \mathbb{R}^{2}$. If $a_{2} \neq 0$, there are three possible phase-portraits depending on the parameter $D$ and they are illustrated in Figure 1. If $D>0$, there are two equilibria in the system (5.2): the saddle point $\left(y^{s}, 0\right)=\left((1+\sqrt{D}) /\left(2 a_{4}\right), 0\right)$ and the center point $\left(y^{c}, 0\right)=\left((1-\sqrt{D}) /\left(2 a_{4}\right), 0\right)$ (they correspond to $\mathcal{H}_{1}=h_{s}$ and $\mathcal{H}_{1}=h_{c}$ respectively $)$. The center point $\left(y^{c}, 0\right)$ is surrounded by periodic orbits with period

$$
T=2 \int_{y_{\circ}^{-}}^{y_{\circ}^{+}} \frac{1}{\sqrt{h-\frac{1}{2} y^{2}+\frac{1}{2} a_{2} I_{\circ} y+\frac{1}{3} a_{4} y^{3}}} d y,
$$

where $y_{\circ}^{-}$and $y_{\circ}^{+}$are found by solving $\mathcal{H}_{1}=h$ for $y$ if $p_{y}=0$. If $h_{s}>h_{c}$ then $h_{c}<h<h_{s}$. Orbits in which $\mathcal{H}_{1}>h_{s}$ are unbounded ${ }^{1}$. There exists a homoclinic connection: the component of $\mathcal{H}_{1}^{-1}\left(h_{s}\right) \cup\left(y^{s}, 0\right)$ which forms a closed curve.

To translate this back to the full normalized system, we take the cross product with the $S^{1}$-action generated by $X_{I_{0}}$. At $\mathcal{H}_{1}=h_{c}$, we have the stable periodic solution of the form $\left(\sqrt{2 I_{0}} \cos \left(\left(1-\varepsilon a_{2} y^{c}\right) t+\varphi_{0}\right), y^{c}, \sqrt{2 I_{\circ}} \sin \left(\left(1-\varepsilon a_{2} y^{c}\right) t+\varphi_{\circ}\right), 0\right)$. The periodic solutions of (5.2) which are found if $h_{c}<h<h_{s}$, produce quasi-periodic solutions in the full normalized system. They live in two dimensional tori in $\mathbb{R}^{4}$ which are the bounded component of $\mathcal{H}_{1}^{-1}(h)$ cross product with $S^{1}$. At $\mathcal{H}_{1}=h_{s}$, we have the unstable periodic solution of the form $\left(\sqrt{2 I_{\circ}} \cos \left(\left(1-\varepsilon a_{2} y^{s}\right) t+\varphi_{\circ}\right), y^{s}, \sqrt{2 I_{\circ}} \sin \left(\left(1-\varepsilon a_{2} y^{s}\right) t+\varphi_{\circ}\right), 0\right)$. This periodic solution has two dimensional unstable and stable manifolds which intersect transversally at the

\footnotetext{
${ }^{1}$ We note that the converse does not hold since $\mathcal{H}_{1}{ }^{-1}(h)$ might have two disconnected components.
} 


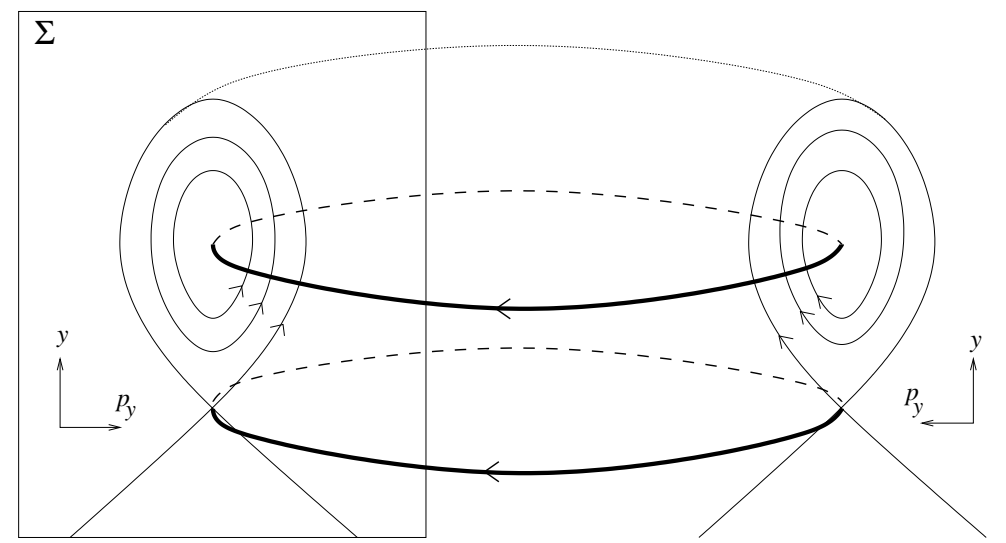

Figure 2: An illustration for the geometry of the phase space of system (5.1) up to $O(\varepsilon)$ in the case where $D>0$ in (5.3). In this picture, the value of $I$ is fixed. The thick lines represent the periodic orbits: the upper one is stable and the lower one is unstable. The unstable periodic solution is connected to itself by a 2-dimensional homoclinic manifold. In the two dimensional Poincare section $\Sigma$, the dynamics is determined by (5.2).

periodic orbit, and are also connected to each other to form a two dimensional manifold in $\mathbb{R}^{4}$ homoclinic to the periodic solution. See Figure 2 for illustration.

Let us consider the cases where $D=0$ in equation (5.3) (we still assume $a_{2} \neq 0$ ). There is only one equilibrium in the system $(5.2)$, that is $\left(1 / 2 a_{4}, 0\right)$. The linearized system of (5.2) has double zero eigenvalues correspond to a saddle-center bifurcation. As the center point and the saddle point of (5.2) coincide, the domain where the solutions are bounded vanishes. For $D<0$, the situation is almost the same as the case where $D=0$ except that there is no equilibrium in the system (5.2). For the full normalized system, the geometry of the phase-portrait for both $D=0$ and $D<0$ can be achieved by taking the cross product of the corresponding phase-portrait in Figure (1) with $S^{1}$.

Let $a_{2}=0$. The system in (5.2) decouples up to $O(\varepsilon)$ and the sign of $D$ in the equation (5.3) is positive. We need only to note the existence of infinitely many periodic orbits of period $T$ where $T / 2 k \pi \in \mathbb{N}$ filling up a two dimensional torus (depending on the period) in phase-space. As we include the higher order terms in the normal form, these periodic solutions become quasi-periodic. The existence of the invariant manifold homoclinic to the periodic solution $\left(S_{1} \times \mathcal{H}_{1}^{-1}(0)\right)$ is not affected by the fact that $a_{2}=0$.

Another degenerate case occurs when $a_{4}=0$ (think of the $y$-symmetric Hamiltonian). In this case, the system (5.2) has one equilibrium for a fixed value of $I_{\circ}$. The eigenvalues of this equilibrium are purely imaginary, i.e. $\pm i$; all solutions of $(5.2)$ are $2 \pi$-periodic. We 
shall discuss this in the next section.

\section{Second order averaging if $a_{4}=0$}

Let $a_{4}=0$. Then the Hamiltonians (4.4) and (4.5) reads

$$
\begin{aligned}
& \mathcal{H}_{1}=\frac{1}{2}\left(\left(y-a_{2} I\right)^{2}+p_{y}{ }^{2}\right)-\frac{1}{2} a_{2} I^{2} \\
& \mathcal{H}_{2}=\frac{1}{2}\left(\frac{5}{6} a_{1}{ }^{2}+\frac{3}{4} b_{1}\right) I^{2}+\left(\frac{1}{2} a_{2}{ }^{2}+a_{1} a_{3}+b_{3}\right) y^{2} I+\frac{1}{4}\left(2 a_{3}{ }^{2}+b_{5}\right) y^{4},
\end{aligned}
$$

with corresponding equations of motion (after rescaling time)

$$
\begin{aligned}
& \frac{d y}{d \tau}=p_{y} \\
& \frac{p y}{d \tau}=-y+a_{2} I+\varepsilon\left(2\left(\frac{1}{2} a_{2}^{2}+a_{1} a_{3}+b_{3}\right) y I+\left(2 a_{3}^{2}+b_{5}\right) y^{3}\right),
\end{aligned}
$$

which is the reduced system for a fixed value of $I$. We first look for the equilibrium of (6.1). Consider the equation

$$
y^{3}+\gamma_{1} y+\gamma_{2}=0
$$

where

$$
\gamma_{1}=\frac{-1+\varepsilon\left(a_{2}^{2}+2 a_{1} a_{3}+2 b_{3}\right) I}{\varepsilon\left(2 a_{3}^{2}+b_{5}\right)} \text { and } \gamma_{2}=\frac{a_{2} I}{\varepsilon\left(2 a_{3}^{2}+b_{5}\right)} .
$$

Using Cardano's formula, we have the discriminant

$$
D_{3}=\left(\gamma_{2} / 2\right)^{2}+\left(\gamma_{1} / 3\right)^{3} .
$$

For (6.1) we have one, two or three equilibria. If $D_{3}>0,(6.2)$ has one real root. $D_{3}=0$ gives two real roots while $D_{3}<0$ corresponds to three real roots. We describe the phaseportrait of (6.1) in Figure 3. There are three different regions in the parameter space $\gamma_{1}-\gamma_{2}$ corresponding to how many equilibria (6.1) has. Depending on the stability of each equilibrium, we have several possibilities for the phase-portraits.

The stability of these equilibria can be derived as following. Let $y_{0}$ be a simple root of the equation (6.2) and write $\mu=2 a_{3}{ }^{2}+b_{5}$. If $\mu>0$, then $\left(y_{0}, 0\right)$ is a stable equilibrium. On the other hand if $\mu<0$, then $\left(y_{0}, 0\right)$ is a saddle point of the system (6.1). If $y_{0}$ is a double root, then $\left(y_{0}, 0\right)$ is an unstable equlibrium.

Since the Hamiltonian of the system (6.1) is a quartic function, one can conclude that, if (6.1) has one or three equilibria, then all of them should correspond to simple roots of (6.2). If it has two equilibria, then one of them corresponds to a simple root while the other is a double root. From this analysis, we can derive the stability of each of the equilibria that exist in the system (6.1). We summarize this in Figure 3.

Remark 6.1 In the case where $\mu=2 a_{3}^{2}+b_{5}=0$, the normalized system is again degenerate, in the sense that the dynamics is nothing but rotation around an elliptic equilibrium. We should then normalize to even higher degree. We expect to have more equilibria compared to those we found for the non-degenerate case. Apart from that, we expect no more complications. 

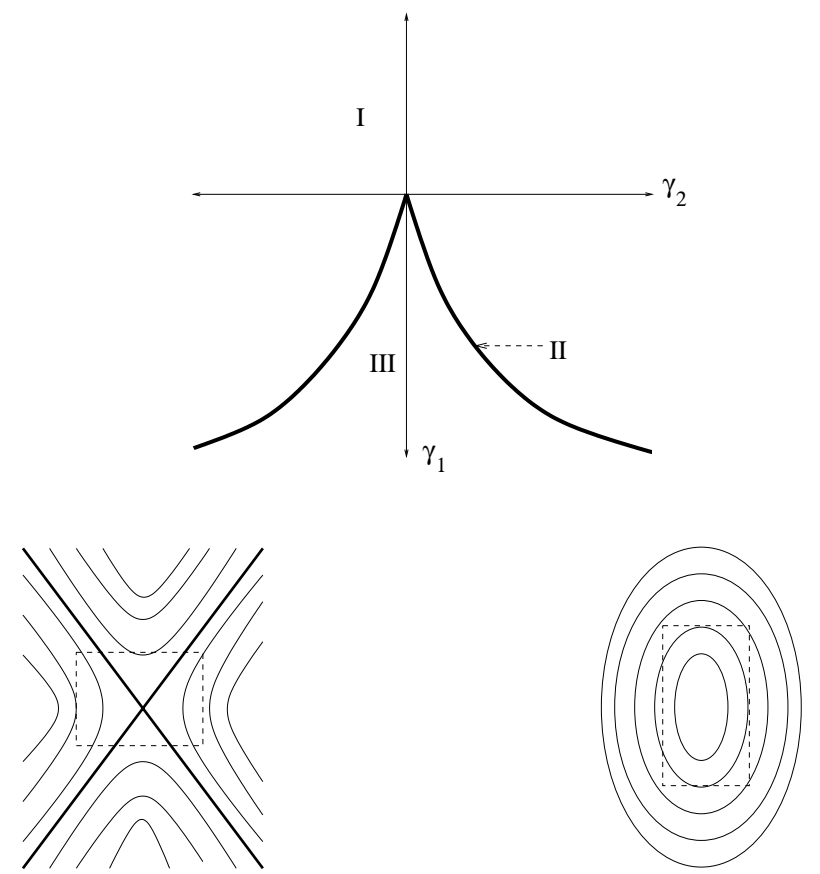

II
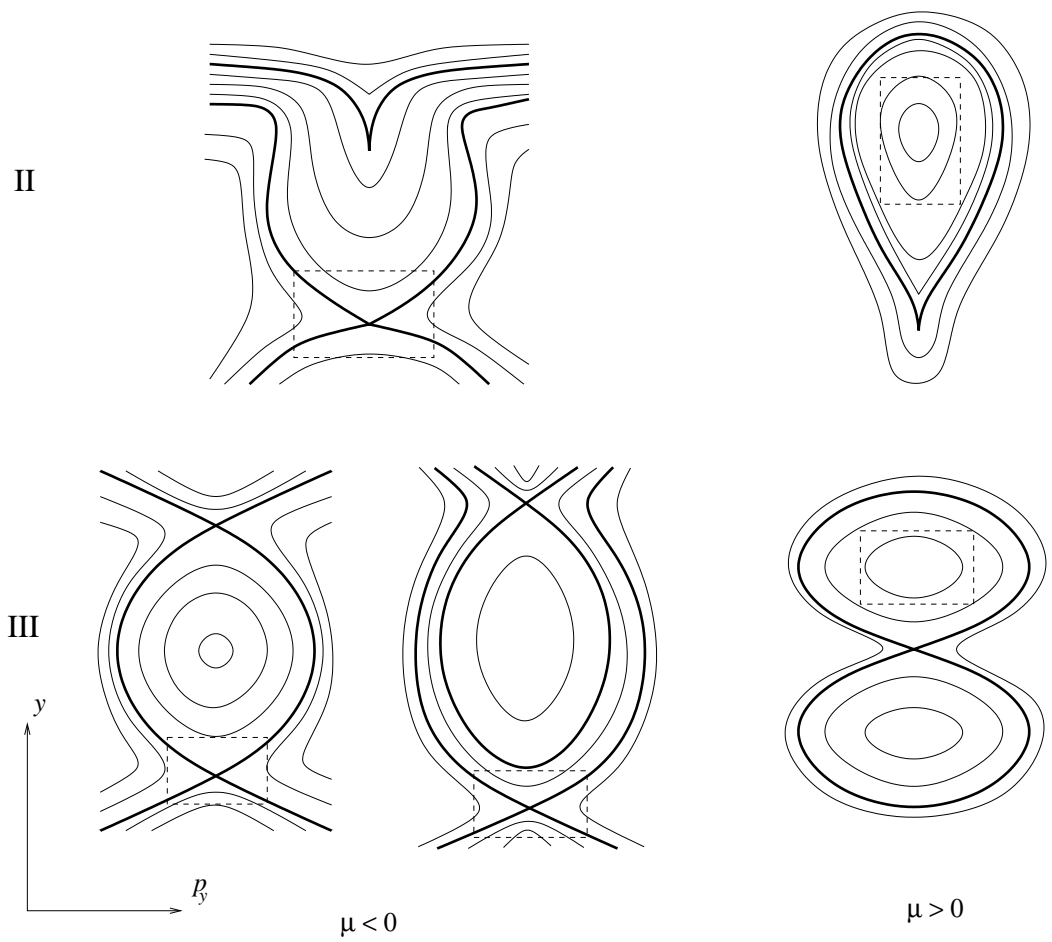

Figure 3: Bifurcation diagram of the system (6.1). The curve depicted in this figure at the top is the curve $\left(\gamma_{2} / 2\right)^{2}+\left(\gamma_{1} / 3\right)^{3}=0$. We draw the possible phase-portraits of the system (6.1) below this bifurcation diagram. For each region (indicated by the Roman numbers I, II, and III), we have two possibilities depending on the sign of $\mu=2 a_{3}^{2}+b_{5}$. The dashed box indicates the location in the reduced space where the normal form is a good approximation of the full system. 


\section{Remark 6.2 Notes on locations of the equilibria and their bifurcation}

One can see that both of $\gamma_{1}$ and $\gamma_{2}$ are $O(1 / \varepsilon)$. This implies that some of the equilibria found in this analysis might also be $O(1 / \varepsilon)$ and this analysis might not be applicable since it is far away from the domain where the normal form is a good approximation of the system. In Figure 3 we place a dashed box around a particular equilibrium in the phaseportraits to indicate the domain where the normal form is a good approximation of the system.

As stated in the last part of the previous section, in the case where $a_{4}=0$ the first order analysis shows that the system has only one equilibrium. This equilibrium can be continued to a equilibrium in the system (6.1) (the one inside the box). During this continuation (by implicit function theorem) the stability of this equilibrium will not change.

Another way of looking at the bifurcation is the following. It is clear that $\gamma_{1} \neq 0$ since $a_{j}$ and $b_{j}$ are independent of $\varepsilon$. This fact excludes the possibility of deforming the cubic equation (6.2) so that it has a single root with multiplicity three.

Remark 6.3 It should be clear that these equilibria of the reduced system (if they survive), correspond to periodic orbits in the full system by taking the cross product of the equilibrium $\left(y_{0}, 0\right)$ with $S^{1}$. The stability of these periodic orbits is the same as in the reduced system. Thus Figure 3 also serves as the bifurcation diagram for the periodic orbit in the full normalized system.

\section{Application of the KAM theorem}

The celebrated Kolmogorov-Arnold-Moser (KAM) theorem is one of the most important theorems in perturbation theory of Hamiltonian dynamics. This theorem relates the dynamics from the normal form analysis to the dynamics in the full system, under some non-degeneracy condition. The theorem itself can be stated in a very general way (see [1] or [3]). As noted previously, the assumption on the frequencies implies that we can only guarantee the validity of the normal form in a rather small domain around the origin. However, the normal form of the system displays structurally stable behavior. Using the KAM theorem, we can validate this behavior.

Consider a Hamiltonian system defined in a $2 n$-dimensional, symplectic space $\mathcal{M}$ in the action-angle variables $(\boldsymbol{\phi}, \boldsymbol{J})$ and symplectic form $d \boldsymbol{\phi} \wedge d \boldsymbol{J}$. The Hamiltonian of the system is $H=H_{\circ}(\boldsymbol{J})+\varepsilon H_{1}(\boldsymbol{\phi}, \boldsymbol{J})$. The unperturbed $(\varepsilon=0)$ Hamiltonian system is clearly integrable with integrals $J_{i}, i=1, \ldots, n$ and the equations of motions are

$$
\begin{aligned}
\dot{\boldsymbol{J}} & =0 \\
\dot{\boldsymbol{\phi}} & =\Lambda(\boldsymbol{J}) .
\end{aligned}
$$

The phase space of the system (7.1) is foliated by invariant tori, parameterized by $I_{j}=$ $c_{j}, j=1, \ldots, n$.

The KAM theorem concerns the preservation of these invariant tori as we turn on the (Hamiltonian) perturbation $\varepsilon H_{1}(\boldsymbol{\phi}, \boldsymbol{J})$. The theorem guarantees the preservation of 
a large number of the invariant tori under some non-degeneracy conditions. In applying this fundamental theorem to a general Hamiltonian system with Hamiltonian $H$, one has to find an integrable Hamiltonian which is asymptotically close to $H$. For two degrees of freedom Hamiltonian systems, the truncated normal form is integrable. The asymptotic relation between the original and the truncated, normalized Hamiltonian system is also clear. Thus, it remains to see if the non-degeneracy condition is satisfied.

For general two degrees of freedom Hamiltonian systems in higher order resonance, the non-degeneracy condition is usually satisfied. In contrast, for the extreme type of higher order resonance, this is not a priori the case. The main difficulty is that the unperturbed integrable system is degenerate.

The version of the KAM theorem stated below is applicable to these extreme type of higher order resonance cases. We will follow the discussion in [3].

Consider the Hamiltonian system with Hamiltonian

$$
H=H_{00}\left(J_{1}\right)+\varepsilon H_{01}(\boldsymbol{J})+\varepsilon^{2} H_{11}(\boldsymbol{\phi}, \boldsymbol{J}),
$$

where $\boldsymbol{J}=\left(J_{1}, J_{2}\right)$. The Hamiltonian $(7.2)$ is called properly degenerate if

$$
\frac{\partial H_{00}}{\partial J_{1}} \neq 0 \text { and } \frac{\partial^{2} H_{01}}{\partial J_{2}^{2}} \neq 0 .
$$

The system $H_{00}\left(J_{1}\right)+\varepsilon H_{01}(\boldsymbol{J})$ is called the intermediate system.

Theorem 7.1 In a properly degenerate system, a large subset of the phase-space of the system generated by Hamiltonian (7.2) is filled by invariant tori which are close to the invariant tori of the intermediate system: $\boldsymbol{J}$ is constant.

Remark 7.2 In a properly degenerate, two degrees of freedom Hamiltonian system, the situation is more regular in the sense, for all initial conditions, the values of the action variables remain forever near their initial values. See [3] for details and the reference therein.

Applying Theorem 7.1 to our system, we define the intermediate system as $H=I+\varepsilon \mathcal{H}_{1}$, with $H_{\circ \circ}=I$. The action and angle variables for the one degree of freedom, Hamiltonian system generated by $\mathcal{H}_{1}$ can be calculated using the generating function $S(J, y)=\int_{y_{0}}^{y} p_{y} d y$. Thus we construct a symplectic transformation to bring $\left(y, p_{y}\right)$ to $(J, \phi)$ such that the equations of motion are of the form (7.1). The non-degeneracy condition on "the frequency" $\Lambda(J)$ follows from the fact that the period function (5.4) in the case $D>0$ in (5.3) and $a_{4} \neq 0$, depends nonlinearly on $h_{P}$. Thus, Theorem 7.1 is applicable to our system.

\section{Application to nonlinear wave equations}

In this section we consider applications of the theory of widely spaced frequencies to nonlinear wave equations with initial-boundary values. Many studies have been devoted to 
such problems, see for instance the survey [28], also [4, 13, 14, 25].

In most of these studies the solution of the continuous system is expanded in an orthogonal series and then projected on a finite subspace using the Galerkin truncation method. This results in a finite set of ordinary differential equations. The next step is then to approximate the solution of this finite system by averaging, see for instance [14, 25], or multi-time scale methods, see [4]. Until now the applications have been to low-order resonance cases.

\subsection{Formulation of the problem}

Let $\Omega=[0, \mu \pi]$ and $L$ be a linear, self-adjoint, elliptic differential operator defined on a dense subset $\mathcal{A} \subseteq H_{\circ}(\Omega)$, i.e. $L: \mathcal{A} \rightarrow H_{\circ}(\Omega)$, where $H_{\circ}(\Omega)$ is a Hilbert space with an inner product $\left\langle_{-},-\right\rangle$. Consider the initial-boundary value problem

$$
\begin{aligned}
& v_{t t}-L v=\varepsilon f\left(v, v_{t}, t\right) \\
& v(0, t)=v(\mu \pi, t)=0 \\
& v(x, 0)=\phi(x) \\
& v_{t}(x, 0)=\psi(x),
\end{aligned}
$$

where $\mu \in \mathbb{R}^{+}, 0<\varepsilon \ll 1, v=v(x, t)$ and $f: \mathcal{D} \rightarrow H_{\circ}\left(\mathcal{D} \subseteq H_{1} \times H_{\circ}\right.$, for a Sobolev space $\left.H_{1}\right)$. Important examples to take for $L$ are

$$
\begin{array}{ll}
L_{1}=\frac{\partial^{2}}{\partial x^{2}}, & \text { the perturbed wave equation } \\
L_{2}=\frac{\partial^{2}}{\partial x^{2}}-\mathbb{I}, & \text { the perturbed dispersive wave equation } \\
L_{3}=-\frac{\partial^{4}}{\partial x^{4}}-p^{2} \mathbb{I}, & \text { the perturbed beam equation, }
\end{array}
$$

with $\mathbb{I}$ the identity operator and $p \in \mathbb{N}$. In this paper we will be mainly concerned with the cases $L=L_{1}$ or $L=L_{2}$.

The question of the existence and uniqueness of solutions of problem (8.1) can be settled in a standard way; see for instance for $L=L_{1}$ and $f\left(u, u_{t}, t\right)=u^{3}$ in [14, 25]. In [25], the authors also consider the case where $L=L_{2}$. For $L=L_{3}$ and $f\left(u, u_{t}, t\right)=u^{2}$, the same question is studied in [4]. After applying the Galerkin truncation method an asymptotic solution of (8.1) is constructed.

Let $\lambda_{n}{ }^{2}$ and $V_{n}(x), n=1,2, \cdots$ be the eigenvalues and the corresponding eigenfunctions of $L$. One of the implications of the assumptions on $L$ is that the set of eigenfunctions $\left\{V_{n}, n=1.2 . \cdots\right\}$ form a denumerable, complete set in $\mathcal{A}$. Thus, we can write for the solution $v(x, t)=\sum_{j} a_{j}(t) V_{j}(x)$. Suppose $N$ is a large natural number and consider a finite eigenfunction expansion of $v$, i.e. $v_{N}(x, t)=\sum_{1}^{N} a_{j}(t) V_{j}(x)$. We substitute $u_{N}$ into (8.1) to obtain

$$
\sum_{1}^{N}\left(\ddot{a}_{j}+\lambda_{j}{ }^{2} a_{j}\right) V_{j}=f\left(\sum_{1}^{N} a_{j} V_{j}, \sum_{1}^{N} \dot{a}_{j} V_{j}, t\right)=\bar{f}(x, t) .
$$


Projecting the last equation to the eigenspace generated by $V_{m}, m=1, \cdots, N$ produces for $m=1, \cdots, N$

$$
\ddot{a}_{m}(t)+\lambda_{m}^{2} a_{m}(t)=\left\langle U_{m}, f\right\rangle(t), \quad \forall t .
$$

In $[13,25]$ it was realized that, choosing the initial condition $\phi$ and $\psi$ to be effective only in some of the $N$ modes, does not excite the other modes dramatically on a long time-scale. Thus, the eigenspace acts as an almost-invariant manifold. This observation was analyzed in [15], pp. 23-24, see also [14], where the author studies and proves the asymptotics of the manifold.

In this section we will not discuss the approximation character of the truncation and averaging procedure of wave equations. This can be done, see [28], but it needs a more detailed analysis which falls outside the scope of the present investigation. Our purpose in this section is to project (8.1) on a finite-dimensional space spanned by the eigenfunctions $V_{1}$ and $V_{M}$ of the operator $L$ corresponding to two widely separated eigenvalues (or modes). The projected system generated by $V_{1}$ and $V_{M}($ for $M \in \mathbb{N})$ is

$$
\begin{aligned}
\ddot{a}_{1}+\lambda_{1}{ }^{2} a_{1} & =\varepsilon \bar{f}_{1} \\
\ddot{a}_{M}+\lambda_{M}{ }^{2} a_{M} & =\varepsilon \bar{f}_{M} .
\end{aligned}
$$

We have to choose $M$ such that $\lambda_{1} / \lambda_{M} \ll 1$.

\subsection{Scaling procedures}

We can think of three different scalings applicable to problem (8.1): spatial domain scaling, time scaling, and scaling by localization.

Spatial domain scaling is effected by putting $\bar{x}=x / \mu$ and writing $v(x, t)$ as $u(\bar{x}, t)$. This scaling transforms the spatial domain $\Omega=[0, \mu \pi]$ to $\bar{\Omega}=[0, \pi]$. Obviously, we also have to re-scale the linear operator $L$, the nonlinear function $f$, and the initial conditions $\phi$ and $\psi$. The nonlinear function $f$, and the initial conditions $\phi$ and $\psi$ are transformed as $v(x, t)$ : by writing new functions $\tilde{f}, \tilde{\phi}$ and $\tilde{\psi}$. In general they will depend on the parameter $\mu$. With respect to the operator, spatial domain scaling results in scaling the eigenvalues of the operator. The first and second rescaled operators are $\tilde{L}_{1}=L_{1} / \mu^{2}$ and $\tilde{L}_{2}=L_{1} / \mu^{2}-\mathbb{I}$. Thus, the transformed initial-boundary value problem becomes (we have dropped the bar)

$$
\begin{aligned}
& u_{t t}-\tilde{L} u=\varepsilon \tilde{f}\left(u, u_{t}, t\right) \\
& u(0, t)=u(\pi, t)=0 \\
& u(x, 0)=\tilde{\phi}(x) \\
& u_{t}(x, 0)=\tilde{\psi}(x),
\end{aligned}
$$

where $\tilde{L}$ is $\tilde{L}_{1}$ or $\tilde{L}_{2}$.

The time scaling procedure is done by putting $\tau=\delta_{1}(\varepsilon) t$ where $\delta_{1}$ is an order function. 
Time scaling is usually carried out simultaneously with localization scaling: $\bar{v}=\delta_{2}(\varepsilon) v$ where $\delta_{2}$ is also an order function. It is easy to see that the scaling in $v$ amounts to scaling in the amplitude $a$ and its time derivative.

Our goal is to use these scaling procedures to get widely separated frequencies in system (8.3) with main interest in $L=L_{1}$ or $L=L_{2}$. Consider the situation where $\mu \gg 1$, a large domain. Let $M \in \mathbb{N}$ be sufficiently large such that $M / \mu=O(1)$, compared to $1 / \mu=\delta(\varepsilon) \ll 1$ (the order function $\delta(\varepsilon)$ is to be determined later). Before projecting to a finite dimensional space by Galerkin truncation, we apply the spatial domain scaling which then bring us to (8.4). For the non-dispersive wave equation we have $\lambda_{1}=O(\delta(\varepsilon))$ while $\lambda_{M}=O(1)$, which means we have a system with widely separated frequencies. Since this does not involve the time rescaling, we have the first type of widely separated frequencies. For the dispersive wave equation this approach does not produce a system with widely separated frequencies since $\lambda_{1}=(1+1 / \mu)$ and $\lambda_{M}=(1+M / \mu)$ are both $O(1)$.

If $\mu=1$, spatial domain scaling makes no sense. Again we choose $M \gg 1$, which implies $\lambda_{M} / \lambda_{1} \gg 1$. This case is called the second type of widely separated frequencies in remark 2.1. By rescaling time, $\tau=\lambda_{M} t$, the projected equations of motion become

$$
\begin{array}{cc}
\ddot{a}_{1}+\left(\frac{\lambda_{1}}{\lambda_{M}}\right)^{2} a_{1} & =\frac{\varepsilon}{\lambda_{M}^{2}} \bar{f}_{1} \\
\ddot{a}_{M}+a_{M} & =\frac{\varepsilon}{\lambda_{M}^{2}} \bar{f}_{M},
\end{array}
$$

in both the dispersive and non-dispersive case. This similarity is interesting. However, one can see that the nonlinearity in (8.5) is very small (of order $O\left(\varepsilon / \lambda_{M}{ }^{2}\right)$. The dynamics in this case, if (8.5) is a Hamiltonian system, would be the same as the usual higher order resonance describe in [23, 27].

\subsection{The Hamiltonian equation $u_{t t}-L u=\varepsilon f(u)$}

We shall now consider perturbations such that the wave equation can be put in a Hamiltonian framework. Note that, starting with a Hamiltonian wave equation, it is not trivial that Galerkin truncation again leads to a Hamiltonian system.

Considering $\mathbb{R}^{4}$ as a symplectic space with symplectic form $\mathrm{d} a_{1} \wedge \mathrm{d} b_{1}+\mathrm{d} a_{M} \wedge \mathrm{d} b_{M}$, we suppose that the right-hand side of system (8.3) is such that

$$
\bar{f}_{1}=-\frac{\partial H_{r}}{\partial a_{1}} \text { and } \bar{f}_{2}=-\frac{\partial H_{r}}{\partial a_{M}}
$$

for a function $H_{r}$ (sufficiently smooth). Thus, system (8.3) has Hamiltonian $H$

$$
H=\frac{1}{2}\left(a_{1}^{2}+\lambda_{1}{ }^{2} b_{1}{ }^{2}\right)+\frac{1}{2}\left(a_{M}{ }^{2}+{\lambda_{2}}^{2}{b_{M}}^{2}\right)+\varepsilon H_{r},
$$

(we may have to re-scale $\varepsilon$ ). This Hamiltonian is not in the standard form (2.3). To bring it to the standard form, define a linear symplectic transformation

$$
\mathcal{F}:\left(a_{1}, a_{M}, b_{1}, b_{M}\right)^{T} \longmapsto\left(a_{1} / \sqrt{\lambda_{1}}, a_{M} / \sqrt{\lambda_{2}}, \sqrt{\lambda_{1}} b_{1}, \sqrt{\lambda_{2}} b_{M}\right)^{T} .
$$


This transformation is also known as diagonalization in a Hamiltonian system. For potential problems, the transformation $\mathcal{F}$ transform $H_{r}$ to $\tilde{H}_{r}$ which depends on $\lambda_{1}$ or $\lambda_{2}$. This may become a problem with widely separated frequencies. The coefficients in $\tilde{H}_{r}$ also depend on the small parameter which changes the asymptotic ordering of the nonlinear terms.

In this subsection we will consider the typical perturbation $f(u)=u^{3}$ which corresponds with a potential problem in the classical sense. It will become clear later that the transformation $\mathcal{F}$ simplifies the dynamics.

First consider (8.4) with: $L=L_{1}, \mu \gg 1, \lambda_{1}=1 / \mu$ and $\lambda_{M}=1$. After some computations one obtains that $f(u)=u^{3}$ corresponds to the system (8.3) with the right-hand side functions

$$
\tilde{f}_{1}=\frac{3}{2} a_{1}^{3}+3 a_{1} a_{M}{ }^{2} \text { and } \tilde{f}_{2}=\frac{3}{2} a_{M}{ }^{3}+3 a_{1}{ }^{2} a_{M} .
$$

Transforming by $\mathcal{F}$ the Hamiltonian becomes

$$
H=\frac{1}{2 \mu}\left(b_{1}^{2}+a_{1}^{2}\right)+\frac{1}{2}\left(b_{M}^{2}+a_{M}^{2}\right)-\varepsilon\left(\frac{3}{8} \mu^{2} a_{1}^{4}+a_{M}^{4}+\frac{3}{2} \mu a_{1}^{2} a_{M}^{2}\right) .
$$

Recall that $1 / \mu=\delta(\varepsilon)$. Choosing $\delta(\varepsilon)^{2}=\varepsilon$, we can write (8.8) as (we use $\delta(\varepsilon)$ as the small parameter instead of $\varepsilon$ )

$$
H=\frac{1}{2} \delta(\varepsilon)\left(b_{1}^{2}+a_{1}^{2}\right)+\frac{1}{2}\left(b_{M}^{2}+a_{M}^{2}\right)-\frac{3}{8} a_{1}^{4}+O(\delta(\varepsilon)) .
$$

By rescaling the variables (localization scaling) by $\delta(\varepsilon) a_{j}, \delta(\varepsilon) b_{j}, j=1, M$ (and then rescaling time) we arrive at

$$
H=\frac{1}{2} \delta(\varepsilon)\left(b_{1}^{2}+a_{1}^{2}\right)+\frac{1}{2}\left(b_{M}^{2}+a_{M}^{2}\right)-\frac{3}{8} \delta(\varepsilon)^{2} a_{1}^{4}+O\left(\delta(\varepsilon)^{4}\right) .
$$

The theory in the previous section (for the degenerate case) can be applied to this Hamiltonian.

The situation for $L_{2}, \mu \gg 1, \lambda_{1}=1 / \mu$ and $\lambda_{M}=1$, need not be considered. The spatial domain scaling fails to produce widely separated frequencies. The other case, if $\mu=1$, for both $L=L_{1}$ or $L_{2}$, the same procedure as derived above can be executed. However, looking carefully at (8.5) we can conclude that it behaves as a Hamiltonian system with non resonant frequencies. The reason for this is that, applying the symplectic transformation $\mathcal{F}$ has the effect of pushing some of the terms in the Hamiltonian to higher order in the small parameter.

More in general, the dynamics of this extreme type of higher order resonance for the perturbation function $f(u)$ with $f$ a polynomial in $u$ will also be trivial as in the example discussed here. 


\subsection{The Hamiltonian equation $u_{t t}-L u=\varepsilon h(x) u^{2}$}

We will now consider another type of perturbation of the initial boundary value problem (8.4) by choosing $\tilde{f}\left(u, u_{t}, t\right)=h(x) u^{2}$ with $h(x)$ a sufficiently smooth, odd function, $2 \pi$ periodic in $x$. Fourier decomposition yields $h(x)=\sum_{j} \alpha_{j} \sin (j x)$.

Let $L=L_{1}$ and $\mu \gg 1$. Using this in (8.4) and projecting to the eigenspace as before, we find for the right-hand side of the equations of motion for mode $k$ ( $\delta_{i j}$ is the Kronecker delta)

$$
\begin{aligned}
\bar{f}_{k}=\frac{1}{4} \sum_{1 \leq j \leq i \leq N}^{N-1}\left(2 \delta_{m, j}-\delta_{m, 2 i+j}-\delta_{m,-2 i+j}+\delta_{m, 2 i-j}\right) \alpha_{j} a_{i}{ }^{2}+ \\
\quad \frac{1}{2} \sum_{1}^{N-2}\left(\delta_{m, j-i+k}+\delta_{m,-j+i+k}-\delta_{m, j+i+k}-\delta_{m,-j-i+k}+\delta_{m, j+i-k}\right) \alpha_{j} a_{i} a_{k} .
\end{aligned}
$$

Assuming again that the eigenspace forms an almost invariant manifold, we can isolate two modes from the full eigenfunction expansion.

If $M>(N+1) / 2$, with $N$ sufficiently large, the right-hand sides of the equations of motion (8.3) are

$$
\bar{f}_{1}=\varepsilon\left(3 \gamma_{1} a_{1}^{2}+2 \gamma_{2} a_{1} a_{M}+\gamma_{3} a_{M}^{2}\right) \text { and } \bar{f}_{2}=\varepsilon\left(\gamma_{2} a_{1}^{2}+2 \gamma_{3} a_{1} a_{M}+3 \gamma_{4} a_{M}^{2}\right),
$$

where $\gamma_{1}=\left(\frac{1}{4} \alpha_{1}-\frac{1}{12} \alpha_{3}\right), \gamma_{2}=\left(\frac{1}{4} \alpha_{M}-\frac{1}{8} \alpha_{M-2}-\frac{1}{8} \alpha_{M+2}\right), \gamma_{3}=\left(\frac{1}{4} \alpha_{1}+\frac{1}{8} \alpha_{2 M-1}-\frac{1}{8} \alpha_{2 M+1}\right)$, and $\gamma_{4}=\frac{1}{8} \alpha_{M}$. The system (8.3) together with (8.11) is a Hamiltonian system with Hamiltonian (after applying transformation $\mathcal{F}$ )

$$
H=\frac{1}{2} \delta_{\varepsilon}\left(b_{1}^{2}+a_{1}^{2}\right)+\frac{1}{2}\left(b_{M}^{2}+a_{M}^{2}\right)-\varepsilon\left(\tilde{\gamma}_{1} a_{1}^{3}+\tilde{\gamma}_{2} a_{1}^{2} a_{M}+\tilde{\gamma}_{3} a_{1} a_{M}{ }^{2}+\gamma_{4} a_{M}{ }^{3}\right),
$$

where $\tilde{\gamma}_{1}=\gamma_{1} / \sqrt{\delta_{\varepsilon}^{3}}, \tilde{\gamma}_{2}=\gamma_{2} /\left(\delta_{\varepsilon}\right)$, and $\tilde{\gamma}_{3}=\gamma_{3} /\left(\sqrt{\delta_{\varepsilon}}\right)$ (recall that $\left.\delta_{\varepsilon} \ll 1\right)$.

We have seen before that a cubic perturbation gives us trivial dynamics due to the fact that the transformation $\mathcal{F}$ eliminates the important term, necessary to get nontrivial dynamics. The perturbation that we are considering now depends on the Fourier coefficients $\alpha_{j}$. These coefficients can be used to keep the important terms in our Hamiltonian (8.12). To keep as many terms as possible in (8.12), we assume $\gamma_{1}=\bar{\gamma}_{1} \delta_{\varepsilon}$ and $\gamma_{2}=\overline{\gamma_{2}} \sqrt{\delta_{\varepsilon}}$. Finally, by choosing $\varepsilon=\delta_{\varepsilon}{ }^{2}$, and rescaling the variables (including time) we have the Hamiltonian

$$
\begin{aligned}
H= & \frac{1}{2} \delta_{\varepsilon}\left(b_{1}{ }^{2}+a_{1}^{2}\right)+\frac{1}{2}\left(b_{M}{ }^{2}+{a_{M}}^{2}\right) \\
& -\delta_{\varepsilon}\left(\bar{\gamma}_{1} a_{1}^{3}+{\overline{\gamma_{2}}}_{1} a_{1}^{2} a_{M}+\tilde{\gamma}_{3} a_{1} a_{M}{ }^{2}\right)+O\left(\delta_{\varepsilon} \sqrt{\delta_{\varepsilon}}\right) .
\end{aligned}
$$

The Hamiltonian (8.13) has the form of (2.1) and hence, the analysis in Section 4.4 is applicable to it. Furthermore, compared to the cubic (or polynomial) perturbation cases, the dynamics of the system generated by the Hamiltonian (8.13) is nontrivial.

The choice of a perturbation of the form $h(x) u^{2}$ is not very restrictive; for instance in [4], the authors consider more or less the same type of perturbation. However, to get 
nontrivial dynamics in the case of widely separated frequencies we have to set some of the parameters in $h$ to be small. This is in contrast with the perturbation $f=u^{3}$ where we have not enough parameters to be scaled.

We have mentioned the result in [15] about the asymptotics of the manifolds. The presence of the parameter $\alpha_{j}, j=1, \ldots, N$,can also be used to improve this asymptotic result by setting some of the $\alpha_{j}$ to be very small. Thus, the function $h(x)$ can be viewed as a filter for modes which we do not want to be present in the system.

\section{Remark 8.1 Homoclinic solution of the wave equations}

In section 5 we have studied several possibilities that could arise when we have a Hamiltonian system with widely separated frequencies. In the case of a wave equation with this special quadratic perturbation, the coefficients of the eigenmodes 1 and $M$ fit in with the analysis in section 5. Let us now try to interpret an interesting solution found in section 5 in the wave equations setting.

In section 5, we found a homoclinic orbit for some values of the parameter. Supposed we can choose the parameter in the wave equations such that this homoclinic orbit exists. Recall that the two-modes expansion of the solution can be written as $u(x, t)=a_{1}(t) U_{1}(x)+a_{M}(t) U_{M}(x)$, where $a_{1}$ and $a_{M}$ satisfies a Hamiltonian system with widely separated frequencies.

We conclude that $U$ is a superposition of two periodic wave forms: $U_{1}$ and $U_{M}$. Choosing the initial values at the critical point, we have $a_{1}$ is constant and $a_{M}$ is oscillating periodically. On the other hand at the homoclinic orbit, we see that the superimposed wave forms evolve to the critical positions for both positive and negative time. We note that during the evolution, the amplitude of $a_{M}$ remains constant while the phase is changing.

\section{Concluding remarks}

In this paper we have analyzed a class of two degrees of freedom Hamiltonian systems where the linearized system consists of two harmonic oscillators and one of the characteristic frequencies of these oscillators is of the same magnitude as the nonlinear terms. In general, the dynamics of this system is shown to be significantly different from Hamiltonian systems with the usual higher order resonance. We have shown that although there are no energy interchanges between the degrees of freedom, this system has a nontrivial dynamics.

\section{Comparison with higher order resonance.}

The first thing to note is the time-scale. A generic system with widely separated frequencies, shows an interesting dynamics on the $1 / \varepsilon$ time-scale while in higher order resonances the characteristic time scale is $1 / \varepsilon^{2}$ and higher. This results from one of the oscillators being strongly nonlinear.

The phase-space around the origin of a system with higher order resonance is foliated by invariant tori. In a system with widely separated frequencies, these tori are slightly 
deformed (see Figure 2). Nevertheless, most of these invariant manifolds contain quasiperiodic motions which is analogous to the higher order resonance cases.

A system with widely separated frequencies does not have a resonance manifold which is typical for higher order resonances. However, the phase-space of a system with widely separated frequencies, contains a manifold homoclinic to a hyperbolic periodic orbit (see again Figure 2). This is comparable to the resonance manifold in higher order resonance cases.

The existence of two normal modes in the normal form of Hamiltonian systems in higher order resonance is typical. For the system with widely separated frequencies, this is not true in general. An extra condition is needed. This extra condition eliminates the coupling term between the degrees of freedom from the normal form. Thus, the interaction between the degrees of freedom in the system with widely separated frequencies is weak in the sense there are no energy interactions, but strong in the sense of phase interactions.

Applications to wave equations are analyzed in this paper. We have pointed out the difficulties of having this kind of systems in a generic potential problem. It might be interesting to consider a more general problem, i.e. perturbations of the form $f\left(u, u_{t}, t\right)$ or even $f\left(u, u_{t}, u_{x}, t\right)$. In subsection 8.4 we only studied the first type of widely separated frequencies. For the other type, it can be done in a similar way.

\section{Acknowledgement}

J.M. Tuwankotta wishes to thank Dario Bambusi (Università degli studi di Milano), Richard Cushman (Universiteit Utrecht), George Haller (Brown University), and Bob Rink (Universiteit Utrecht) for stimulating discussions, KNAW and CICAT TUDelft for financial support, and Santi Goenarso for her support in various ways.

\section{References}

[1] Abraham, R., Marsden, J.E., Foundations of Mechanics. The Benjamin/Cummings Publ. Co., Reading, Mass., 1987.

[2] Arnold, V.I., Mathematical Methods of Classical Mechanics, Springer-Verlag, New York etc., 1978.

[3] Arnold, V.I., Kozlov, V.V., Neishtadt, A.I., Mathematical Aspects of Classical and Celestial Mechanics, Encyclopedia of Mathematical Sciences Vol. 3, Dynamical System, V.I. Arnold (ed.), Springer-Verlag, New York etc., 1988.

[4] Boertjens, G.J., van Horssen, W.T., An Asymptotic Theory for a Beam Equations with a Quadratic Perturbation, SIAM J. Appl. Math., vol. 60, pp. 602-632, 2000.

[5] Broer, H.W., Chow, S.N., Kim, Y., Vegter, G., A normally elliptic Hamiltonian bifurcation, ZAMP 44, pp. 389-432, 1993. 
[6] Broer, H.W., Chow, S.N., Kim, Y., Vegter, G., The Hamiltonian Double-Zero Eigenvalue, Fields Institute Communications, vol. 4, pp. 1-19, 1995.

[7] Churchill, R.C., Kummer, M., Rod, D.L., On Averaging, Reduction, and Symmetry in Hamiltonian Systems, Journal of Differential Equations 49, 1983, pp. 359-414.

[8] Cicogna, G. Gaeta,G., Symmetry and Perturbation Theory in Nonlinear Dynamics, Lecture Notes in Physics 57, Springer-Verlag, 1999.

[9] Crommelin, D., Homoclinic Dynamics: a Scenario for Atmospheric Ultra-LowFrequency Variability, in preparation.

[10] Gaeta,G., Poincaré renormalized forms , Ann. Inst. Henri Poincaré, vol. 70, no. 6, 1999, pp. 461-514.

[11] Guckenheimer, J., Holmes, P.H., Nonlinear Oscillations, Dynamical Systems, and Bifurcations of Vector Fields, Applied Math. Sciences 42, Springer-Verlag, 1997.

[12] Haller, G., Chaos Near Resonance, Applied Math. Sciences 138, Springer-Verlag, New York etc., 1999.

[13] Kevorkian, J., Cole, J.D., Multiple Time Scale and Singular Perturbation Methods, Applied Math. Sciences, Springer-Verlag, New York etc., 1996.

[14] Krol, M.S., On a Galerkin-averaging Method for weakly Non-linear Wave Equations, Math. Methods Applied Sciences 11, pp. 649-664, 1989.

[15] Krol, M.S., The Method of Averaging in Partial Differential Equations, Ph.D. Thesis, Universiteit Utrecht, 1990.

[16] Langford, W.F., Zhan, K.,Interactions of Andonov-Hopf and Bogdanov-Takens Bifurcations, Fields Institute Communications, vol. 24,pp. 365-383, 1999.

[17] Langford, W.F., Zhan, K.,Hopf Bifurcations Near 0 : 1 Resonance, BTNA'98 Proceedings, eds. Chen, Chow and Li, pp. 1-18, Springer-Verlag, New York etc., 1999.

[18] Nayfeh, A.H., Mook, D.T., Nonlinear Oscillations, Wiley-Interscience, New York, 1979.

[19] Nayfeh, S.A., Nayfeh, A.H., Nonlinear interactions between two widely spaced modesexternal excitation, Int. J. Bif. Chaos 3, pp. 417-427, 1993.

[20] Nayfeh, A.H., Chin, C.-M., Nonlinear interactions in a parametrically excited system with widely spaced frequencies, Nonlin. Dyn. 7, pp. 195-216, 1995.

[21] Palacián, J., Yanguas, P.,Reduction of Polynomial Planar Hamiltonians with Quadratic Unperturbed Part, SIAM Review, vol 42 number 4, pp. 671-691, 2000. 
[22] Rink, B., Symmetry and resonance in periodic FPU chains, Comm. Math. Phys. 218, Springer-Verlag, pp. 665-685, 2001.

[23] Sanders, J.A., Are higher order resonances really interesting?, Celestial Mech. 16, pp. 421-440, 1978.

[24] Sanders, J.A., Verhulst, F., Averaging Method on Nonlinear Dynamical System, Applied Math. Sciences 59, Springer-Verlag, New York etc., 1985.

[25] Stroucken, A.C.J., Verhulst, F., The Galerkin-Averaging Method for Nonlinear, Undamped Continuous Systems, Math. Meth. in Appl. Sci. 9, pp. 520 - 549, 1987.

[26] van der Burgh, A.H.P., On The Higher Order Asymptotic Approximations for the Solutions of the Equations of Motion of an Elastic Pendulum, Journal of Sound and Vibration 42, pp. 463-475, 1975.

[27] Tuwankotta, J.M., Verhulst, F., Symmetry and Resonance in Hamiltonian System, SIAM J. Appl. Math, vol 61 number 4, pp. 1369-1385, 2000.

[28] Verhulst, F., On averaging methods for partial differential equations, in SPT98Symmetry and perturbation Theory II (A. Degasperis and G. Gaeta, eds.), World Scientific, pp. 79-95, 1999. 